
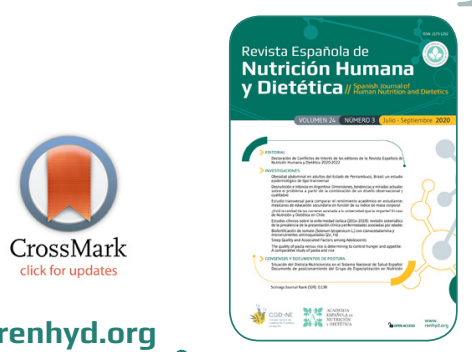

\title{
EDITORIAL
}

\section{Declaración de Conflictos de Interés de los editores de la Revista Española de Nutrición Humana y Dietética 2020-2022}

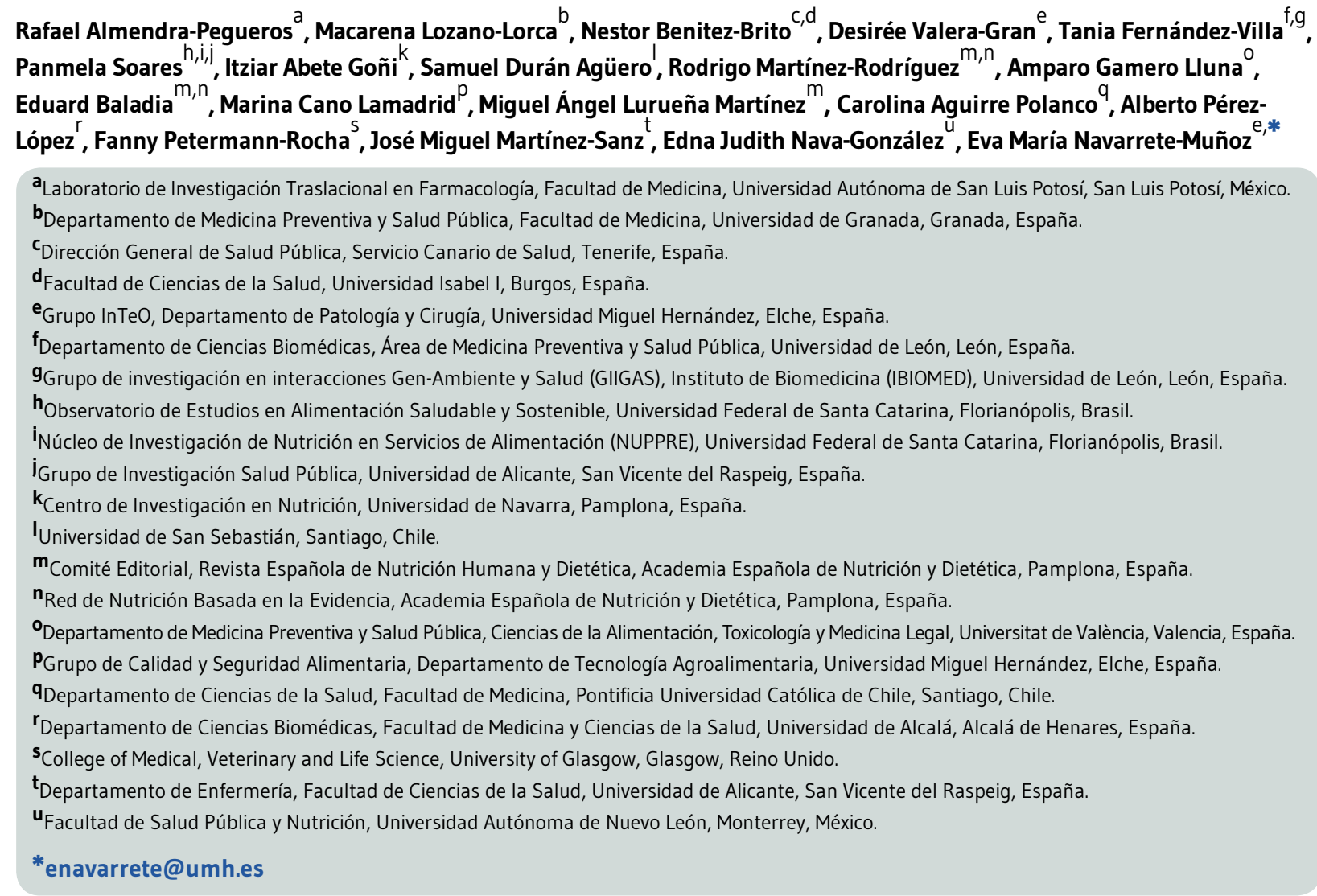

Editor Asignado: Rodrigo Martínez-Rodríguez. Comité Editorial de la Revista Española de Nutrición Humana y Dietética. Pamplona, España.

Recibido el 31 de julio de 2020; aceptado el 2 de agosto de 2020; publicado el 30 de septiembre de 2020.

\section{CITA}

Almendra-Pegueros R, Lozano-Lorca M, Benitez-Brito N, Valera-Gran D, Fernández-Villa T, Soares P, Abete Goñi I, Durán Agüero S, Martínez-Rodríguez R, Gamero Lluna A, Baladia E, Cano Lamadrid M, Lurueña Martínez MA, Aguirre Polanco C, Pérez-López A, Petermann-Rocha F, Martínez-Sanz JM, Nava-González EJ, Navarrete-Muñoz EM. Declaración de Conflictos de Interés de los editores de la Revista Española de Nutrición Humana y Dietética 2020-2022. Rev Esp Nutr Hum Diet. 2020; 24(3): 187-9. doi: 10.14306/renhyd.24.3.1123 
Se reconoce como Conflicto de Interés (COI), al conjunto de condiciones en las que el juicio profesional en relación a un interés primario, tiende a ser indebidamente influenciado por un interés secundario (como por ejemplo el beneficio económico) ${ }^{1}$. Esta situación no es ajena a los procesos editoriales de revistas científicas, estando presente desde la producción de manuscritos por los/as autores/as, la revisión por pares y las gestiones de los/as miembros de los equipos editoriales.

Sin embargo, publicaciones previas han descrito que sólo entre el $30 \%$ y el $40 \%$ de las revistas del área biomédica con revisión por pares, solicitan la declaración de COls al equipo editorial ${ }^{2}$. Este dato contrasta con el hecho de que el $96 \%$ de estas mismas revistas soliciten esta declaración a los/as autores/as asi como a los/as revisores/as s., $^{3,4}$

\section{¿Porqué la declaración de COls por el equipo editorial?}

Es bien sabido que los COls de autores/as pueden influir en los resultados de sus investigaciones, como se ha evidenciado en el caso de la participación de la industria farmacéutica en el diseño de intervenciones con estatinas para la reducción de la lipoproteína de baja densidad ${ }^{5}$, o la participación de la industria alimentaria en en efecto del consumo de bebidas azucaradas sobre la ganancia de peso ${ }^{6}$. Sin embargo, también las personas que integran los comités editoriales pueden presentar potenciales COls, puesto que pueden estar recibiendo, de forma particular o como representante de la revista, beneficios o prebendas de compañías o empresas que pueden influir en el alcance o las temáticas a las que se les da prioridad dentro de la revista7,8. Igualmente los/as integrantes pueden tener otros COls no financieros, como por ejemplo las áreas de interés en este ámbito de investigación, intereses religiosos, intereses políticos, entre otros, que podrían influir a la hora de tomar decisiones editoriales ${ }^{9,10}$.

Por tanto es necesario, que los/as integrantes de los equipos editoriales hagan visibles sus potenciales COls para que los/ as lectores/as, autores/as y revisores/as puedan tenerlos en cuenta cuando reciben una decisión editorial. Estos COls deberían declararse siguiendo las recomendaciones del International Committee of Medical Journal Editors (ICMJE) asentadas en las Recommendations for the Conduct, Reporting, Editing, and Publication of Scholarly work in Medical Journals ${ }^{11}$, así como contribuir en la conservación de la confianza depositada por autores/as, revisores/as y lectores/as de nuestra revista ${ }^{12}$.

\section{¿Qué se debe declarar en los COls? ¿Es o no es un posible COI?}

En la mayoría de los casos, la declaración de COls se encuentra enfocada a situaciones financieras y/o de participación comercial. Sin embargo, hay otros aspectos que no deberían olvidarse especialmente en revistas del ámbito de la nutrición humana y dietética.

En 2018, loannidis y Trepanowski recogían que los intereses personales, como los hábitos y patrones alimentarios o las áreas de investigación, podrían influir de igual forma que los intereses financieros en las investigaciones en este ámbito ${ }^{13}$ Por ello, es necesario ser transparente y recoger la declaración de todos los potenciales COls de los/as autores/as, revisores/as y, cómo no, de los/as editores/as.

Esta propuesta ha generado diversas opiniones ${ }^{14-16}$, motivando la reflexión sobre la mejor forma de declarar los COls en la investigación en dietética y nutrición humana, así como la participación y regulación de la industria de alimentos en los procesos de investigación ${ }^{17}$. Por este motivo, algunas revistas han presentado sus propias perspectivas para el manejo de contribuciones/envíos que hayan sido financiadas por la industria de alimentos ${ }^{18}$.

Ante esta situación, la Revista Española de Nutrición Humana y Dietética ha integrado en estos últimos años diferentes iniciativas con el fin de reducir los potenciales COls como son:

1. El único patrocinador de la revista es la Academia Española de Nutrición Humana y Dietética. No se recibe financiación directa de ningún otro/a patrocinador/a.

2. Más del $75 \%$ de los/as integrantes del equipo editorial no tienen ninguna vinculación con la Academia Española de Nutrición Humana y Dietética.

3. En todos los manuscritos se declara el editor asociado que se ocupó de la gestión del manuscrito.

4. Los/as autores/as deben declarar sus COls en la plataforma de la revista al enviar el manuscrito como se indica en la guía para autores/as (http://renhyd. org/index.php/renhyd/pages/view/norm) y en las políticas de la revista (http://renhyd.org/index.php/ renhyd/about/editorialPolicies\#custom-9).

5. Los/as revisores/as deben declarar sus COls en la plataforma de la revista al enviar su revisión como se indica en la guía para autores/as (http://renhyd.org/ index.php/renhyd/pages/view/normreferees) y en las políticas de la revista (http://renhyd.org/index.php/ renhyd/about/editorialPolicies\#custom-9).

$Y$ en aras de seguir contribuyendo a esta transparencia en la declaración del COls se ha decidido actualizar los perfiles de los/as editores/as en la página web de la revista (http:// renhyd.org/index.php/renhyd/about/editorialTeam) incluyendo un cuadro de declaración de conflictos de interés 
de cada uno de los/as integrantes del equipo. $Y$ además se ha decidido realizar un resumen de los potenciales COls de cada integrante del equipo editorial que podrá revisar en: http://www.renhyd.org/index.php/renhyd/pages/view/ editorCOIs.

\section{CONFLICTO DE INTERESES}

Los autores son editores de la Revista Española de Nutrición Humana y Dietética.

\section{REFERENCIAS}

(1) Thompson DF. Understanding financial conflicts of interest. N Engl J Med. 1993; 329(8): 573-6.

(2) Bosch X, Pericas JM, Hernández C, Doti P. Financial, nonfinancial and editors' conflicts of interest in high-impact biomedical journals. Eur ] Clin Invest. 2013; 43(7): 660-7.

(3) Resnik DB, Elmore SA. Conflict of Interest in Journal Peer Review. Toxicol Pathol. 2018; 46(2): 112-4.

(4) Resnik DB, Konecny B, Kissling GE. Conflict of Interest and Funding Disclosure Policies of Environmental, Occupational, and Public Health Journals. J Occup Environ Med. 2017; 59(1): 28-33.

(5) Naci H, Dias S, Ades AE. Industry sponsorship bias in research findings: a network meta-analysis of LDL cholesterol reduction in randomised trials of statins. BMJ. 2014; 349: g5741.

(6) Mandrioli D, Kearns CE, Bero LA. Relationship between
Research Outcomes and Risk of Bias, Study Sponsorship, and Author Financial Conflicts of Interest in Reviews of the Effects of Artificially Sweetened Beverages on Weight Outcomes: A Systematic Review of Reviews. PLoS ONE. 2016; 11(9).

(7) Liu J], Bell CM, Matelski J], Detsky AS, Cram P. Payments by US pharmaceutical and medical device manufacturers to US medical journal editors: retrospective observational study. BMJ. 2017; 359: j4619.

(8) Haque W, Minhajuddin A, Gupta A, Agrawal D. Conflicts of interest of editors of medical journals. PLoS ONE. 2018; 13(5).

(9) Easley TJ. Medical Journals, Publishers, and Conflict of Interest. JAMA. 2017; 317(17): 1759-60.

(10) Romain PL. Conflicts of interest in research: looking out for number one means keeping the primary interest front and center. Curr Rev Musculoskelet Med. 2015; 8(2): 122-7.

(11) ICMJE | Recommendations [Internet]. [citado 31 de julio de 2020]. Disponible en: http://www.icmje.org/ recommendations/

(12) Garza C, Stover PJ, Ohlhorst SD, Field MS, Steinbrook R, Rowe $S$, et al. Best practices in nutrition science to earn and keep the public's trust. Am J Clin Nutr. 2019; 109(1): 225-43.

(13) loannidis JPA, Trepanowski JF. Disclosures in Nutrition Research: Why It Is Different. JAMA. 2018; 319(6): 547-8.

(14) Ludwig DS, Kushi LH, Heymsfield SB. Conflicts of Interest in Nutrition Research. JAMA. 2018; 320(1): 93.

(15) Bero L, Grundy Q. Conflicts of Interest in Nutrition Research. JAMA. 2018; 320(1): 93-4.

(16) Navarrete-Muñoz EM, Tardón A, Romaguera D, MartínezGonzález MÁ, Vioque J. La financiación de la industria alimentaria y la investigación epidemiológica sobre nutrición y salud. Gac Sanit. 2018; 32(2): 168-71.

(17) Mozaffarian D. Conflict of Interest and the Role of the Food Industry in Nutrition Research. JAMA. 2017; 317(17): 1755-6.

(18) Soares MJ, Müller MJ, Boeing H, Maffeis C, Misra A, Muscogiuri $G$, et al. Conflict of interest in nutrition research: an editorial perspective. Eur ] Clin Nutr. 2019; 73(9): 1213-5. 